Paper presented at: Nuclear Structure at the Extremes, Lewes, Sussex, UK, June 16-19 1998. To be published in J. Phys. G: Nucl. Part. Phys.

\title{
Core excitation in halo nucleus breakup
}

\author{
J A Tostevin \\ Department of Physics, School of Physical Sciences, University of Surrey, \\ Guildford, Surrey GU2 5XH, United Kingdom
}

\begin{abstract}
We estimate the magnitudes of core excited state cross sections, following halo nucleus dissociation on a light target. Calculations of integrated partial cross sections for the ${ }^{9} \mathrm{Be}\left({ }^{11} \mathrm{Be},{ }^{10} \mathrm{Be} \gamma\right)$ reaction at $60 \mathrm{MeV} /$ nucleon are presented and some sensitivities to inputs discussed. Reasonable agreement is obtained with preliminary experimental estimates for this system.
\end{abstract}

\section{Introduction}

A conventional experimental arrangement for the study of halo nuclei has been to detect a heavy fragment, with the mass and charge of the core of the halo nucleus, following the dissociation of the incident beam. So, in the case of a ${ }^{11} \mathrm{Be}$ beam, ${ }^{10} \mathrm{Be}$ fragments are detected with velocities close to that of the beam velocity. The measured cross sections for the detection of this heavy fragment, both total and differential, have been used to attempt to clarify the halo nucleus structure, for example in analyses of the widths of the core momentum distribution [1]. These usually assume a dominance of the cross section corresponding to the halo particle removal from the core (ground) state. Recent experiments, such as discussed here by Hansen, in which decay photons from excited states of the heavy core fragment are identified, would indicate that, potentially, a significant contribution to these measured core distributions arises from excited and not ground state core configurations.

A preliminary analysis of measurements of the ${ }^{9} \mathrm{Be}\left({ }^{11} \mathrm{Be},{ }^{10} \mathrm{Be} \gamma\right)$ reaction with a 60 $\mathrm{MeV} /$ nucleon ${ }^{11} \mathrm{Be}$ beam [2] is shown as the experimental points in Figs. 1 and 3. It is consistent with about $25 \%$ of the cross section going to the $1^{-}(5.960 \mathrm{MeV})$ and $2^{-}$ $(6.263 \mathrm{MeV})$ excited core states while the cross section to the $2^{+}(3.368 \mathrm{MeV})$ state is consistent with zero.

\section{Eikonal model calculations of nuclear breakup}

To provide a first theoretical orientation, eikonal model calculations are carried out for both the diffraction dissociation and stripping [3] contributions to the integrated partial cross sections for a particular ${ }^{10} \mathrm{Be}^{*}$ core state $c$ following ${ }^{11} \mathrm{Be}$ breakup. For the calculation of each final state cross section $\sigma(c)$ the projectile ground state $\left|\phi_{I M}\right\rangle$ is described as a valence neutron, with single particle quantum numbers $(n j \ell)$ and 
an associated spectroscopic factor, with respect to the specific core state. Such configurations are written $\left|\phi_{I M}^{c}\right\rangle$, with $I$ the intrinsic spin and $M$ the spin projection of the ground state. These component cross sections will be computed from the integrals over the projectile c.m. impact parameter $\mathbf{b}$,

$$
\begin{aligned}
\sigma(c, \text { diff }) & =\frac{1}{2 I+1} \int d \mathbf{b}\left[\sum_{M}\left\langle\left.\phi_{I M}^{c}||\left(1-S_{c} S_{n}\right)\right|^{2} \mid \phi_{I M}^{c}\right\rangle\right. \\
& \left.-\sum_{M M^{\prime}}\left|\left\langle\phi_{I M^{\prime}}^{c}\left|\left(1-S_{c} S_{n}\right)\right| \phi_{I M}^{c}\right\rangle\right|^{2}\right],
\end{aligned}
$$

and

$$
\sigma(c, \text { strip })=\frac{1}{2 I+1} \int d \mathbf{b} \sum_{M}\left\langle\left.\phi_{I M}^{c}\left|\left(1-\left|S_{n}\right|^{2}\right)\right| S_{c}\right|^{2} \mid \phi_{I M}^{c}\right\rangle .
$$

The $S_{c}$ and $S_{n}$ are the profile functions $[4,5]$ for the core and neutron, functions of their own impact parameters, determined by the assumed core- and neutrontarget interactions. While the diffractive dissociation and stripping contribution are comparable for weakly bound (n-halo) states, the stripping contribution dominates in the case of non-s-wave and more tightly bound neutron removal - as for excited core states. Clearly only $\sigma(c$, diff $)$ is sensitive to the refractive components of the core and $\mathrm{n}$-target interactions and so details of the real parts of the interactions are of little importance when $\sigma(c$, strip) dominates. The core-target interaction is in any case highly absorptive at the energies of interest and $S_{c}$ has often been replaced (without loss of accuracy) by a sharp cutoff (black disk) form.

$\sigma(c$, strip) on the other hand is sensitive, through the $\mathrm{n}$ absorption probability $\left(1-\left|S_{n}\right|^{2}\right)$, to the assumed geometry and absorptive character of the n-target interaction, a sensitivity which is assessed qualitatively in the following.

\section{Application to ${ }^{11}$ Be breakup}

We estimate partial cross sections $\sigma(c)$ to the $0^{+}, 2^{+}, 1^{-}$, and $2^{-}{ }^{10} \mathrm{Be}$ final states in the ${ }^{9} \mathrm{Be}\left({ }^{11} \mathrm{Be},{ }^{10} \mathrm{Be} \gamma\right)$ reaction at $60 \mathrm{MeV} /$ nucleon. The $0^{+}$and $2^{+}$states are assumed to arise from dissociation/stripping of the $\left(2 s_{\frac{1}{2}} \otimes 0^{+}, S_{n}=0.504 \mathrm{MeV}\right)$ neutron halo, and the $\left(1 d_{\frac{5}{2}} \otimes 2^{+}, S_{n}=3.872 \mathrm{MeV}\right)$ excited core configurations, respectively, in the ${ }^{11} \mathrm{Be}$ (g.s.). The spectroscopic factors for these two configurations, in both the shell model [6] and simple excited core cluster models [7], are essentially in agreement. We take $\mathrm{S}\left(2 s_{\frac{1}{2}} \otimes 0^{+}\right)=0.74$, and $\mathrm{S}\left(1 d_{\frac{5}{2}} \otimes 2^{+}\right)=0.20[6]$.

The excited $J^{\pi}=1^{-}(5.96 \mathrm{MeV})$ and $2^{-}(6.26 \mathrm{MeV}){ }^{10} \mathrm{Be}^{*}$ final states are considered as arising from the removal of a tightly bound $\left(1 p_{\frac{3}{2}} \otimes J^{\pi}, S_{n} \approx 6.5 \mathrm{MeV}\right)$ neutron. These states are themselves less than $1 \mathrm{MeV}$ from the ${ }^{9} \mathrm{Be}+\mathrm{n}$ breakup threshold $(6.8 \mathrm{MeV})$ and might be thought of as resulting from the stripping of a $1 p_{\frac{3}{2}}$ neutron from the ${ }^{10} \mathrm{Be}\left(0^{+}\right)$ core of the ${ }^{11} \mathrm{Be}($ g.s. $)$ - producing a $\left[2 s_{\frac{1}{2}} \otimes{ }^{9} \mathrm{Be}\left(\frac{3}{2}^{-}\right)\right] 1^{-}$or $\left[2 s_{\frac{1}{2}} \otimes{ }^{9} \mathrm{Be}\left(\frac{3}{2}^{-}\right)\right] 2^{-}$halo-like ${ }^{10} \mathrm{Be}^{*}$ core. This same nucleon stripping mechanism was used previously to study the unbound ${ }^{10} \mathrm{Li}$ system [8], in that case by the removal of a proton from the ${ }^{10} \mathrm{Be}$ core. 
The spectroscopic factors for the $1^{-}$and $2^{-}$configurations in the ${ }^{11} \mathrm{Be}(\mathrm{g} . \mathrm{s}$.$) are$ estimated as $\mathrm{S}\left(1 p_{\frac{3}{2}} \otimes 1^{-}\right)=0.69$ and $\mathrm{S}\left(1 p_{\frac{3}{2}} \otimes 2^{-}\right)=0.58[6]$. For the $\frac{1}{2}^{+}{ }^{11}$ Be case we have an additional simplification in Eqs. (1) and (2), and

$$
\left\langle\phi_{I M^{\prime}}^{c} \mid \phi_{I M}^{c}\right\rangle_{\text {spins }}=\left[u_{n j \ell}^{2}(r) / 4 \pi\right] \delta_{M M^{\prime}}
$$

where $u_{n j \ell}$ is the neutron radial wave function. All neutron single particle states are calculated in a central Woods-Saxon well with radius and diffuseness parameters (1.15 $\mathrm{fm}, 0.5 \mathrm{fm}$ ). This calculates a ${ }^{11} \mathrm{Be}$ (g.s.) with rms radius of $2.90 \mathrm{fm}$, assuming a ${ }^{10} \mathrm{Be}$ (g.s.) rms radius of $2.28 \mathrm{fm}[4]$.

Cross sections are calculated from Eqs. (1), (2) and (3). The profile functions for the core- and n-target $\left({ }^{9} \mathrm{Be}\right)$ systems are calculated using the optical limit of Glauber theory [4]. We assume a finite-range NN formfactor $f_{N N}$ (see Eqs. (2) and (3) of [5]), free nn and $n p$ cross sections, real to imaginary ratios of the forward scattering amplitude due to Ray [9] (at $100 \mathrm{MeV}$ ) and a Gaussian finite range parameter $\beta_{n n}=\beta_{n p}=0.5 \mathrm{fm}$. Using this NN formfactor, and matter densities with rms radii consistent with electron scattering, we reproduce the measured $\sigma_{R}\left({ }^{12} \mathrm{C}+{ }^{12} \mathrm{C}\right)$ and $\sigma_{R}\left({ }^{12} \mathrm{C}+{ }^{27} \mathrm{Al}\right)$ at $83 \mathrm{MeV} /$ nucleon [10] and calculate $\sigma_{R}\left(\mathrm{p}+{ }^{9} \mathrm{Be}\right)=29.1 \mathrm{fm}^{2}$ at $60 \mathrm{MeV} /$ nucleon, consistent with experiment [11]. Calculations using these profile functions are denoted $\mathrm{NN}$ in the following.

The calculated $\sigma(c$, diff $), \sigma(c$, strip), and their sum, before multiplication by the spectroscopic factors, are shown in Figure 1 when using the NN based profile functions. The dominance of the stripping term, for other than the $0^{+}$halo core state, is evident. The full curve shows the summed cross sections multiplied by the stated spectroscopic factors and is in reasonable agreement with the trends of the preliminary data for this system.

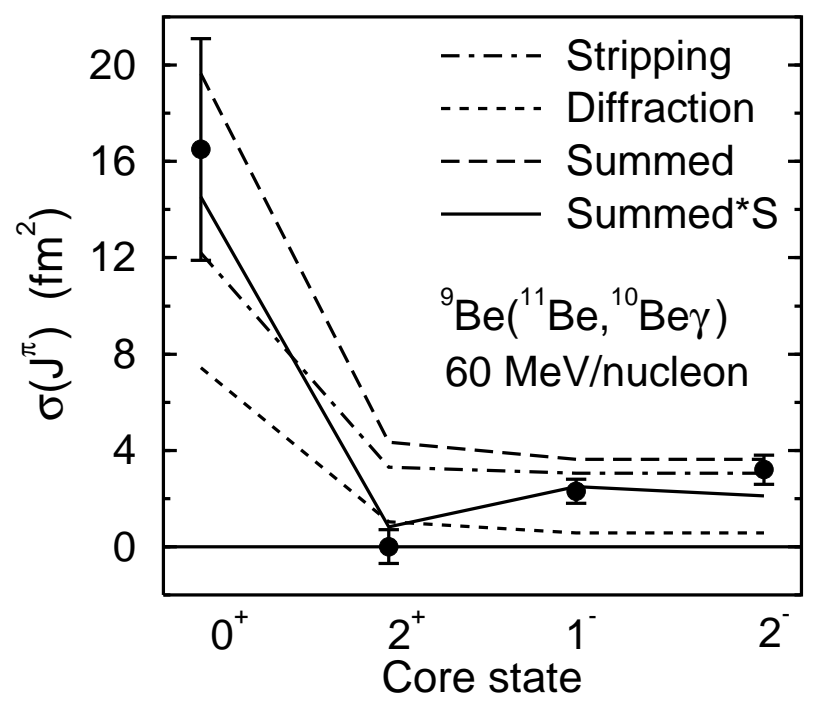

Figure 1. Calculated and deduced $\sigma(c)$ for the indicated ${ }^{10} \mathrm{Be}^{*}$ core states in the ${ }^{9} \mathrm{Be}\left({ }^{11} \mathrm{Be},{ }^{10} \mathrm{Be} \gamma\right)$ reaction at $60 \mathrm{MeV} /$ nucleon, calculated using the $\mathrm{NN}$ description of the profile functions. 
In the case of the $1^{-}$and $2^{-}$final states the calculation of the core profile function $S_{c}$ assumed the ${ }^{10} \mathrm{Be}^{*}$ core state to be an extended $\left[2 s_{\frac{1}{2}} \otimes{ }^{9} \mathrm{Be}\left(\frac{3}{2}^{-}\right)\right]$halo-like entity, with $S_{n}=0.5 \mathrm{MeV}$ and wave function $\varphi$, thus approximating the removal of a $p_{\frac{3}{2}}$ neutron from the ${ }^{10} \mathrm{Be}\left(0^{+}\right)$core of the ${ }^{11} \mathrm{Be}($ g.s. $) . S_{c}$ is then calculated to include breakup of this composite core [4], as

$$
S_{c}(b)=\left\langle\varphi\left|S_{9} S_{n}\right| \varphi\right\rangle,
$$

with the ${ }^{9} \mathrm{Be}$-target profile function $S_{9}$ calculated assuming a ${ }^{9} \mathrm{Be}$ rms radius of $2.28 \mathrm{fm}$.

As expected, in these cases with a fragile halo-like core state, the core survival probability is reduced compared to calculations which assume a normally sized ${ }^{10} \mathrm{Be}$ core. The cross sections are 10-15\% smaller when using $S_{c}$ given by Eq. (4) rather than the $S_{c}$ used for the ground state. The calculations presented in the Figures are based on the use of Eq. (4).

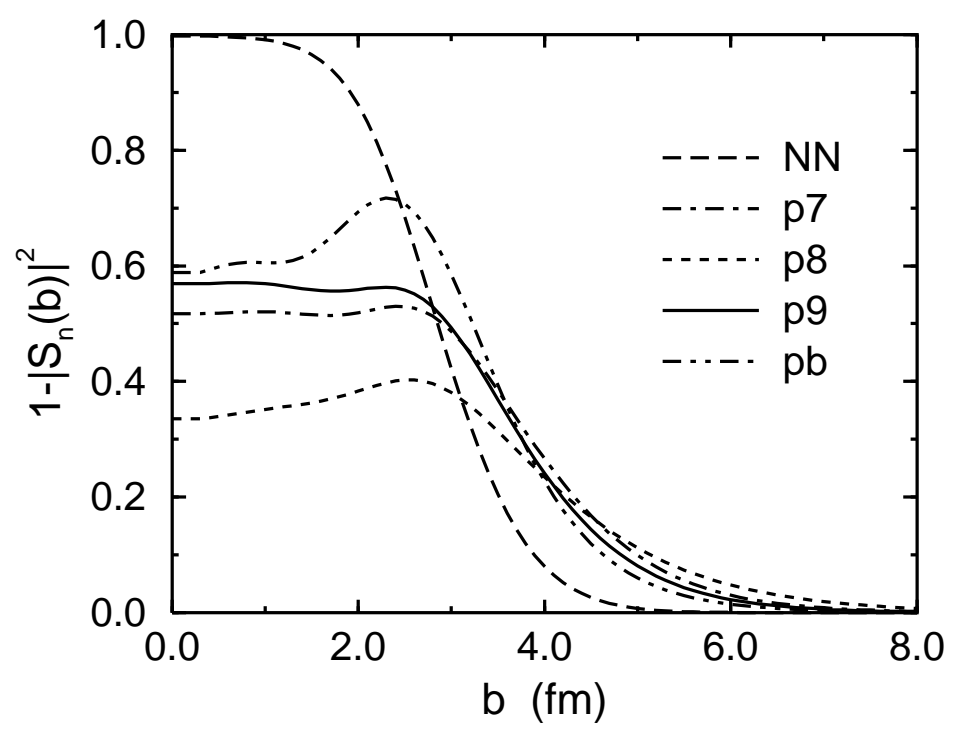

Figure 2. Calculated $n$ absorption probability $\left(1-\left|S_{n}\right|^{2}\right)$ as a function of impact parameter from phenomenological optical potential and optical limit NN descriptions of the $n-{ }^{9}$ Be profile function.

To assess the sensitivity of calculations to the assumed $n-{ }^{9}$ Be interaction, referred to earlier, $S_{n}$ is also calculated using the phenomenological nucleon $-{ }^{9}$ Be optical potentials 7,8 and 9 of [12] and (b) of [13]. The qualitatively very different inputs to the stripping calculations which result, through the $\left(1-\left|S_{n}\right|^{2}\right)$ factor, are shown in Figure 2 for these phenomenological and the NN profile function cases. The less volume and more surface absorptive nature of the phenomenological interactions is evident and it is clear that this theoretical/experimental input will be a very important one - in particular for calculations of the absolute magnitudes of the stripping cross sections.

The averages of the cross sections computed with these phenomenological $S_{n}$ are compared with those using the optical limit NN description in Figure 3. The calculated cross sections are different typically by a factor of two in cases where the stripping 


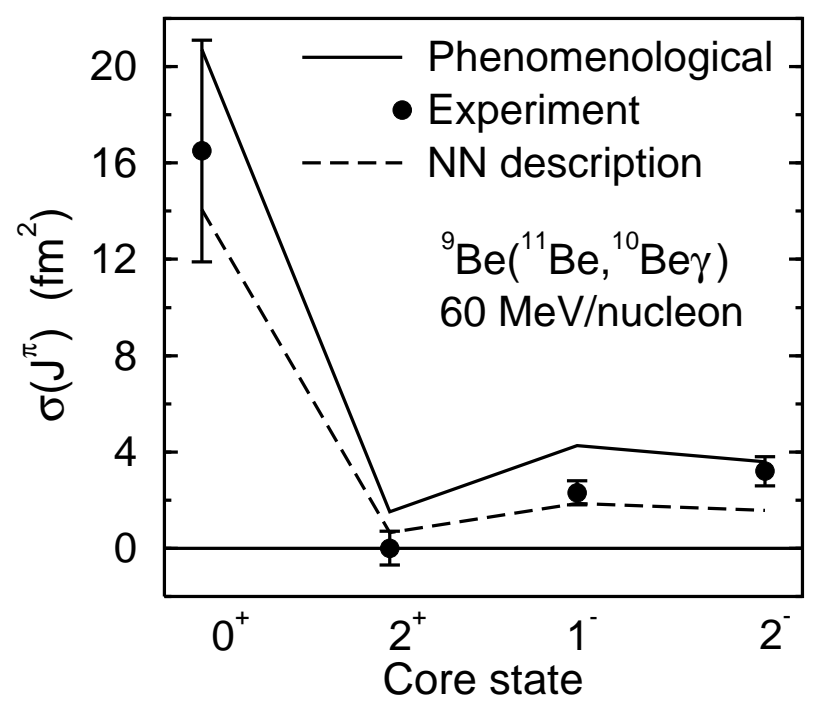

Figure 3. Calculated and deduced $\sigma(c)$ for the indicated ${ }^{10} \mathrm{Be}^{*}$ core states in the ${ }^{9} \mathrm{Be}\left({ }^{11} \mathrm{Be},{ }^{10} \mathrm{Be} \gamma\right)$ reaction at $60 \mathrm{MeV} /$ nucleon. Calculations use the phenomenological and the $\mathrm{NN}$ descriptions of the $\mathrm{n}-{ }^{9}$ Be profile function.

cross section is dominant. The two very different descriptions of the profile function do however encompass the preliminary data and the trends, and the relative sizes of the halo state and core excited cross sections, in the two cases are very similar. The summed calculated cross sections are 30 and $18 \mathrm{fm}^{2}$, respectively, compared to the experimental estimate of $22 \mathrm{fm}^{2}$.

Other applications, to test the consistency of such model calculations against the still rather limited experimental data for other projectile systems, are in progress and will be discussed elsewhere $[2,14]$.

\section{Summary}

The magnitudes of integrated core excited state cross sections following halo nucleus dissociation on a light target are estimated within the eikonal approximation. We describe model calculations of partial cross sections in the case of the ${ }^{9} \mathrm{Be}\left({ }^{11} \mathrm{Be},{ }^{10} \mathrm{Be}^{*}\right)$ reaction at $60 \mathrm{MeV} /$ nucleon which provide a reasonable qualitative description of preliminary experimental information for this system. These calculations support the possibility that a significant fraction of measured core fragment cross sections arise from excited core configurations in the final state.

\section{Acknowledgments}

The financial support of the United Kingdom Engineering and Physical Sciences Research Council (EPSRC) in the form of Grant No. GR/J95867 is gratefully 
acknowledged. The author would like to acknowledge numerous stimulating discussions with Prof. P. Gregers Hansen on the work presented.

\section{References}

[1] Orr N A 1997 Nucl. Phys. A 616 155c, presents a recent review

[2] Chartier M et al, Michigan State University, to be published

[3] Barranco F, Vigezzi E and Broglia R A 1996 Z. Phys. A 356 45, and references therein

[4] Al-Khalili J S, Tostevin J A and Thompson I J 1996 Phys. Rev. C 541843

[5] Tostevin J A and Al-Khalili J S 1997 Nucl. Phys. A 616 418c

[6] Brown B A and Wildenthal B H 1988 Ann. Rev. Nucl. Sci. 38 191; Brown B A 1995 Proc. Int. Conf. Exotic Nuclei and Atomic Masses (ENAM-95), Arles, France, eds. de Saint-Simon M and Sorlin O (Editions Frontières, Gif-sur-Yvette, 1996) 451; and to be published

[7] Nunes F M, Thompson I J and Johnson R C 1996 Nucl. Phys. A 596171

[8] Zinser M et al 1995 Phys. Rev. Lett. 751719

[9] Ray L 1979 Phys. Rev. C 201857

[10] Kox S et al 1987 Phys. Rev. C 351678

[11] Renberg P U et al 1972 Nucl. Phys. A 18381

[12] Mani G S 1971 Nucl. Phys. A 165145

[13] Clarke N M et al 1970 Nucl. Phys. A 157145

[14] Alahari N et al 1998 Contribution to ENAM 98, Bellaire, Michigan, 23-27 June 1998, to be published 